\title{
Navigation System for Ground Vehicles using Temporally Interconnected Observers
}

\author{
Pierre-Jean Bristeau, Nicolas Petit
}

\begin{abstract}
In this paper, we propose an analysis of a recently introduced navigation technique for an automotive vehicle. This method involves several observers, each designed for a particular type of trajectory, that are turned on and off according to a switching policy. Each observer takes advantage on the observability properties found along these trajectories. The contribution of this paper is an analysis of the convergence properties of the resulting temporal interconnection of observers. In details, decay rate estimates of each of the observers, arising from their uniform complete observability properties, are used to guarantee convergence of the constituted navigation system.
\end{abstract}

\section{INTRODUCTION}

Lately, an attempt was made to propose a theoretical formalism for common practices in the field of observer design of vehicular systems. A typical example of such practices $^{1}$ is found in the navigation systems of cruise and guided missiles as is thoroughly detailed in [3], [4], [5]. In such applications, several distinct phases of the system trajectory are considered to reconstruct, sequentially through distinct algorithms, subsets of the whole state vector of the system. Practically, an initialization using attitude measurements from an inertial measurement unit (IMU) is performed (at rest) on the launch platform, then, after the boost phase and during mid-course (high speed) flight, measurements made by the IMU and additional knowledge of the corresponding motions from terrain navigation radars is used to determine in-flight alignment of inertial sensors, and, finally, the (maneuvering) transition to terminal target is performed through data fusion from other additional sources of information such as optical devices. Decomposing the trajectory in distinct phases during which the system has well recognizable dynamics eases the design of data fusion algorithms as it suggests relatively easy and dependable means to reconstruct certain variables during certain phases. The various components of the state of the system are not estimated all at once, but, instead, well defined subsets are reconstructed during each particular phase of the vehicle trajectory.

For the problem under consideration in this paper, one can follow a similar strategy. We desire to estimate the state of

P.J. Bristeau is $\mathrm{PhD}$ candidate in Mathematics and Control, CAS, Unité Mathématiques et Systèmes, MINES ParisTech, 60 bvd Saint-Michel, 75272 Paris, FRANCE pierre-jean.bristeau@mines-paristech. fr

N. Petit is with the CAS, Unité Mathématiques et Systèmes, MINES ParisTech, 60 bvd Saint-Michel, 75272 Paris, FRANCE

${ }^{1}$ Other examples can be found in navigation algorithms using the ZUPT technique [1], [2] a ground vehicle by means of a navigation system incorporating an IMU, a velocimeter and an altimeter, combined in a data fusion algorithm. We consider the vehicle as a six degrees of freedom (DOF) rigid body moving without sideslip. The vehicle is characterized by a tridimensional position, a curvilinear velocity and three attitude angles. Certainly, the list of considered sensors, which excludes GPS, is not sufficient to reconstruct the absolute position and heading of the vehicle, but this is not our goal. Only relative positioning is desired here.

A key feature of the setup we study in this paper is the availability of a velocimeter which provides a relatively dependable estimate of the vehicle body velocity. This sensor is commonly available in vehicles today. It can be obtained through various sensor technologies (e.g. Doppler radar [6], camera [7], Pitot tube), each technology having its own flaws and advantages [8]. Yet, this scalar information is not sufficient to estimate the motion of the vehicle as rotations come into play. Gyroscopes can be used to determine rateof-turn information, but they also have (non-constant) biases which are causes of substantial drifts.

In [9], a method analyzing the observability along various possible trajectories of the system was proposed. Investigations have served to prove the theoretical asymptotic convergence of a collection of Kalman filters used to estimate the states of linear time-varying (LTV) dynamics. The convergence of each individual Kalman filter was proven under the assumption of Uniform Complete Observability (UCO) which, itself, was related to an usual differential rank test of observability. Separately but simultaneously, the velocity, the angular dynamics (angles, rates and biases), and finally, the accelerations (and the biases associated to the sensors) were estimated. Combining observers in the previously discussed way follows along lines similar to the classic observers interconnection theory (see [10]).

As discussed in [9], and briefly recalled in this paper, the main difficulty is to estimate the angular dynamics of the vehicle. For this problem, we propose another level of interconnection, named temporally interconnected observers (TIO) which we consider arguing that, during straight-line motion one can estimate the pitch dynamics, while, during curve motion, it is possible to catch the roll dynamics, and that, eventually, yaw bias can be estimated at rest).

A natural, but so-far overlooked, question is the convergence of this TIO scheme. Indeed, having separate subsets of the state variables estimate errors decay during distinct (non overlapping) periods does not automatically guarantee the convergence of the full state to actual values. The culprit 
is that the remaining variables must be propagated without any possibility of corrections when they are not observed. Careful investigations reveal that, in our case, the TIO can provide convergence under certain simple assumptions on the switching policy. Establishing this result is the main contribution of this paper.

The paper is organized as follows. In Section II, we detail the vehicle navigation problem under consideration. We expose the general TIO structure proposed in [9], and state a problem of convergence involving a succession of straightline and curve motions. In Section III, we establish the UCO property of the system (focusing on curve motion) and draw some conclusions on the estimatability (as defined in [11]) of the system. The main argument of proof is the study of a time-varying pulsation oscillator appearing in the attitude dynamics. In Section IV, we establish the convergence of the proposed TIO. Here, the estimates established earlier serve to guarantee the convergence of a discrete-time dynamics governing the system according to the switching policy. This TIO strategy is currently used on-board a prototype navigation system. In Section V, we present results of an actual implementation.

\section{INTERCONNECTIONS OF OBSERVERS FOR GROUND VEHICLE NAVIGATION}

\section{A. Description of the vehicle navigation problem}

We consider a vehicle which is equipped with a velocimeter, three gyroscopes and an altimeter. The velocimeter measures the (curvilinear) velocity of the vehicle. The gyroscopes are biased and the inputs of the driver are unknown.

The variable $u$ denotes the longitudinal velocity of the vehicle, $p, q, r$ are the rotation speeds, $\phi, \theta, \psi$ are the roll, pitch and yaw angles, and $z$ is the altitude (as is illustrated in Fig. 1). The subscript $m$ indicates the corresponding measurement, and $b_{p}, b_{q}, b_{r}$ are the bias of the gyroscopes which are (slowly) varying over time. The complete state to be estimated is

$$
\left[\begin{array}{lllllllllll}
u & z & \phi & \theta & \psi & b_{p} & b_{q} & b_{r} & p & q & r
\end{array}\right]^{T}
$$

The dynamics and measurement equations of the $6 \mathrm{DOF}$ rigid body are derived under the assumptions of zero sideslip, and small angles of roll and pitch. The dynamics of

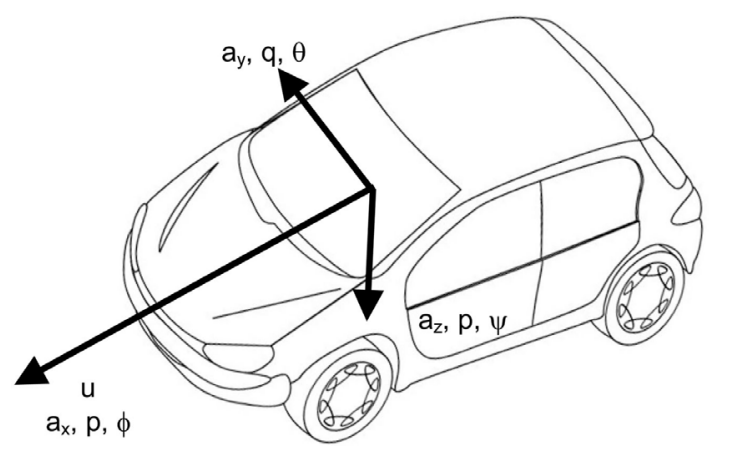

Fig. 1. Notations in the body frame

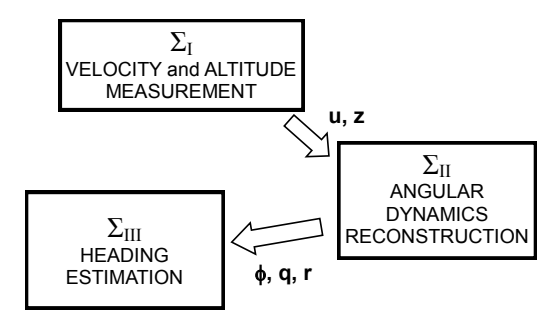

Fig. 2. Cascaded estimation

the rotation speeds are unknown. Dependable differential equations that can be written are

$$
\left\{\begin{aligned}
\dot{z}(t) & =-u(t) \theta(t), & & \dot{\phi}(t)=p(t)+r(t) \theta(t), \\
\dot{\theta}(t) & =q(t)-r(t) \phi(t), & & \dot{\psi}(t)=r(t)+q(t) \phi(t)
\end{aligned}\right.
$$

Finally, the measurement equations are

$$
\begin{cases}u_{m}(t)=u(t), & z_{m}(t)=z(t), \\ p_{m}(t)=p(t)-b_{p}(t), & q_{m}(t)=q(t)-b_{q}(t), \\ r_{m}(t)=r(t)-b_{r}(t) & \end{cases}
$$

\section{B. Two levels of interconnection}

The method we study to estimate the state (1) is inspired by the theory of observers for interconnected systems (widely detailed in [10]). To simplify the following discussion, rather than considering the velocity $u$ and the altitude $z$ in the state and in the measurement, we consider $\theta_{m}$ a virtual measurement of $\theta$ which can be obtained by $\theta_{m}=-\dot{\hat{z}} / \hat{u}$. The estimation of the subset $X_{I}=\left[\begin{array}{ll}u & z\end{array}\right]^{T}$ can be isolated in one first problem consisting in a velocity and altitude observer directly derived from the velocimeter and the barometer. Considering the velocity and the altitude as known parameters, a second problem is to estimate the biases of the gyroscopes, the pitch and roll angles, and the corresponding rates of turn. From Eq. (3), the estimates $\hat{p}$ and $\hat{q}$ can be reconstructed from the measurements $p_{m}$ and $q_{m}$ and the biases estimates $\hat{b}_{p}$ and $\hat{b}_{q}$. So, the subset of (1) under study in the second problem is $\left[\begin{array}{llllll}\phi & \theta & \psi & b_{p} & b_{q} & b_{r}\end{array}\right]^{T}$. Finally, we assume that all the preceding variables are known parameters, and we reconstruct the heading rate of turn and compute the heading (third problem). This observer design corresponds to the cascade interconnection pictured in Fig. 2. The three problems detailed above corresponds to subsystems $\Sigma_{I}, \Sigma_{I I}$ and $\Sigma_{I I I}$ describing, respectively, the velocity measurement, the angular dynamics reconstruction, and the heading estimation.

Establishing separately (i.e. when the parameters are known) the observability of the velocity, and the observability of yaw rate (first and third problems) is trivial, so one shall focus on the second problem and determine how to estimate, in a sequence, the reduced state $\left[\begin{array}{llllll}\phi & \theta & \psi & b_{p} & b_{q} & b_{r}\end{array}\right]^{T}$.

If the velocity is null, the vehicle is at rest, biases are obtained by direct reading of the gyrometers measurements. In particular, $b_{r}$ is estimated. Leaving out the goal to estimate $\psi$ without any absolute heading reference, one is left with the estimation of the reduced state $\mathbf{X}_{I I}=\left[\begin{array}{llll}\phi & \theta & b_{p} & b_{q}\end{array}\right]^{T}$ which is the subject of the next section. 


\section{Dynamics under consideration}

We now wish to write the dynamics of $\mathbf{X}_{I I}$. If the yaw rate is null, the vehicle is going in straight-line else the vehicle is in a curve. After leaving the rest phase, the vehicle switches between these last two types of trajectory. Along the trajectories, the dynamics are as follows

$$
\begin{aligned}
& \text { Straight-line motion } \\
& \text { Curve motion } \\
& \left\{\begin{array}{l}
\dot{\phi}=p=p_{m}+b_{p} \\
\dot{\theta}=q=q_{m}+b_{q} \\
\dot{b}_{p}=0, \quad \dot{b}_{q}=0
\end{array}\right. \\
& \left\{\begin{array}{l}
\dot{\phi}=\left(p_{m}+b_{p}\right)+r \theta \\
\dot{\theta}=\left(q_{m}+b_{q}\right)-r \phi \\
\dot{b}_{p}=0, \quad \dot{b}_{q}=0
\end{array}\right.
\end{aligned}
$$

For sake of simplicity, biases, which are in fact slowly varying, are considered constant in the equations. It shall be noted that having variable biases does not interfere in the observability analysis, but complexifies the convergence study, e.g. by requiring extra states to be modeled.

To study the dynamics in straight line and curve motion, we define matrices $\left(A_{1}, B_{1}, C_{1}\right)$ and $\left(A_{2}, B_{2}, C_{2}, D_{2}\right)$ such that, with $\mathbf{X}_{1}=\left[\begin{array}{ll}\theta & b_{q}\end{array}\right]^{T}, \mathbf{X}_{2}=\left[\begin{array}{lll}\phi & \theta & b_{p}\end{array}\right]^{T}$ and $\mathbf{U}=\left[\begin{array}{ll}p_{m} & q_{m}\end{array}\right]^{T}$, the previous equations (4) yield

$$
\begin{aligned}
& \left\{\begin{aligned}
\dot{\mathbf{X}}_{1} & =A_{1} \mathbf{X}_{1}+B_{1} \mathbf{U} \\
& =\left(\begin{array}{ll}
0 & 1 \\
0 & 0
\end{array}\right) \mathbf{X}_{1}+\left[\begin{array}{ll}
0 & 1 \\
0 & 0
\end{array}\right] \mathbf{U} \\
\mathbf{Y}_{1} & =C_{1} \mathbf{X}_{1}=\left(\begin{array}{ll}
1 & 0
\end{array}\right) \mathbf{X}_{1}
\end{aligned}\right. \\
& \left\{\begin{aligned}
\dot{\mathbf{X}}_{2} & =A_{2}(t) \mathbf{X}_{2}+B_{2} \mathbf{U}+D_{2} b_{q} \\
& =\left(\begin{array}{ccc}
0 & r & 1 \\
-r & 0 & 0 \\
0 & 0 & 0
\end{array}\right) \mathbf{X}_{2}+\left[\begin{array}{ll}
1 & 0 \\
0 & 1 \\
0 & 0
\end{array}\right] \mathbf{U}+\left[\begin{array}{l}
0 \\
1 \\
0
\end{array}\right] b_{q} \\
\mathbf{Y}_{2} & =C_{2} \mathbf{X}_{2}=\left(\begin{array}{lll}
0 & 1 & 0
\end{array}\right) \mathbf{X}_{2}
\end{aligned}\right.
\end{aligned}
$$

Leaving out the cases when the vehicle is at rest, we consider a sequence of straight lines and curve motions. Let us note $T_{1}$ the straight-line motion duration, $T_{2}$ the curve motion duration. It is assumed that all the straight-lines have the same duration and that the same holds for straight lines. This simplification is only made for ease of notations and can be easily relaxed. Recursively, with $i \geq 0$ and $t_{0}=0$, define $t_{i}$ the straight-line motion starting time $\left(t_{i+1}=t_{i}+T_{1}+T_{2}\right)$, $\chi(2 \mathrm{i})$ the variable $\chi$ at time $t_{i}, \chi(2 \mathrm{i}+1)$ the variable $\chi$ at time $t_{i}+T_{1}$. The times $t_{i}$ are known and defined by the switching policy. This policy is not the subject of study here. ${ }^{2}$

\section{The proposed TIO structure}

The TIO algorithm we propose involves two observers $\hat{\mathbf{X}}_{1}$ and $\hat{\mathbf{X}}_{2}$, which each provides an asymptotically converging estimate of $\mathbf{X}_{1}$ and $\mathbf{X}_{2}$ which are subsets of $\mathbf{X}_{I I}$. Existence and convergence of these observers is proven in Section III. The two observers are used alternatively according to the

\footnotetext{
${ }^{2}$ An example of switching policy is, when the estimated yaw rate is null, to use the straight-line model, and otherwise, to use the curve model. The value of $\hat{r}$ depends on the estimation of the yaw gyrometer bias, but potential error on $\hat{b}_{r}$ does not undermine the TIO algorithm due to the small value of the bias. On the contrary, the switching policy to/from rest is more critical and, therefore, the velocimeter needs to be very accurate because at rest, biases are directly updated from the gyrometers which can be detrimental. A vehicle during a parking maneuver at low-speed can generate interpretation errors and result in wrong estimates for biases.
}

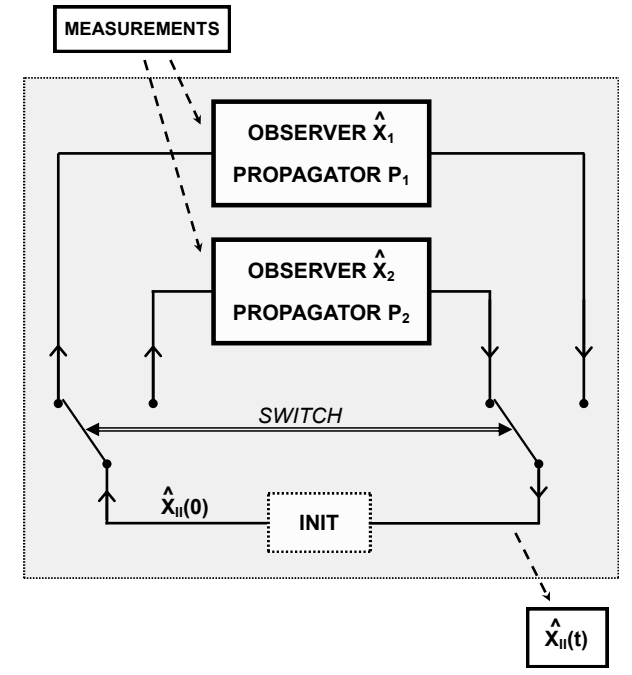

Fig. 3. Temporally interconnected observers (TIO) structure for estimating the vehicle angular dynamics.

switching policy. The continuity between the observers is achieved by a propagator $P_{i}$ of the unestimated states along with the observer $X_{i}$. This structure is illustrated in Fig. 3 where the block INIT represents the initialization which occurs at every switching time. The newly switchedon observer-propagator is initialized using the estimates obtained from the other one. The whole TIO structure can be considered as an observer which provides an estimate $\hat{\mathbf{X}}_{I I}$ from the measurements.

Between the times $t_{i}$ and $t_{i}+T_{1}$ (straight-line motion), the following observer-propagator is considered.

$$
\begin{aligned}
& \text { Observer: } \hat{\mathbf{X}}_{1} \text { is used to estimate } \mathbf{X}_{1}=\left[\begin{array}{ll}
\theta & b_{q}
\end{array}\right]^{T} \\
& \text { Propagator: } P_{1}\left\{\dot{\phi}=p_{m}+b_{p}, \quad \dot{b}_{p}=0\right\}
\end{aligned}
$$

Respectively, between the times $t_{i}+T_{1}$ and $t_{i}+T_{1}+T_{2}$ (curve motion), the other observer-propagator is used.

$$
\begin{aligned}
& \text { Observer: } \hat{\mathbf{X}}_{2} \text { is used to estimate } \mathbf{X}_{2}=\left[\begin{array}{lll}
\phi & \theta & b_{p}
\end{array}\right]^{T} \\
& \text { Propagator: } P_{2}\left\{\dot{b}_{q}=0\right\}
\end{aligned}
$$

The question we now wish to address is the asymptotic behavior of the estimate $\hat{\mathbf{X}}_{I I}$ as the vehicle travels along a succession of straight-lines and curves.

\section{OBSERVABILITY AND ESTIMATABILITY}

Now, the two subsystems (5)-(6) observability properties are studied. Prior to these investigations, we briefly recall some key results on such linear time-varying (LTV) systems that serve to bound estimation errors of candidate observers.

\section{A. Recall on Uniform Complete Observability (UCO)}

Consider a (multivariable) LTV system, with $A(t), B(t), C(t)$ analytic

$$
\dot{x}(t)=A(t) x(t)+B(t) u(t), \quad y(t)=C(t) x(t)
$$

Note $\Phi(s, t)$ the transition matrix associated to $\mathrm{A}$,

$$
\frac{\partial \Phi}{\partial t}(t, s)=A(t) \Phi(t, s), \quad \Phi(t, t)=I
$$


and $W^{*}\left(t_{0}, t\right)$ the reconstructibility Grammian

$$
W^{*}\left(t_{0}, t\right)=\int_{t_{0}}^{t} \Phi^{T}(s, t) C^{T}(s) C(s) \Phi(s, t) d s
$$

Definition 1: [?][12] The system (9) is uniformly completely observable (UCO) if the following relations hold for all $t$ :

$$
0<\alpha_{0}(\sigma) I \leq W^{*}(t-\sigma, t) \leq \alpha_{1}(\sigma) I
$$

$0<\alpha_{2}(\sigma) I \leq \Phi^{T}(t-\sigma, t) W^{*}(t-\sigma, t) \Phi(t-\sigma, t) \leq \alpha_{3}(\sigma) I$

where $\sigma$ is a fixed positive constant, and $\alpha_{i}(\sigma)$ is used to denote a constant solely determined by its argument.

In the case of bounded matrices (with the same notations as above), the following theorem provides a simpler necessary and sufficient condition.

Theorem 1: [13][14] A bounded system $[A(t), B(t), C(t)]$ is $U C O$ if and only if there exists $\sigma>0$ such that for all $t$,

$$
W^{*}(t-\sigma, t) \geq \alpha_{0}(\sigma) I>0
$$

\section{B. Recall on Uniform Complete Estimatability (UCE)}

Definition 2: [11] The system (9) is said to be uniformly completely estimatable (UCE) if, for any pair of real numbers $m$ and $M$ such that $m \leq M$, there are positive numbers $\delta$, $\eta$ and an estimator gain $K(\cdot)$ such that any solution of the following system

$$
\dot{\tilde{\mathbf{X}}}(t)=(A(t)-K(t) C(t)) \tilde{\mathbf{X}}(t)
$$

with

$$
\left\{\begin{array}{l}
\tilde{X}(t)=\hat{X}(t)-X(t) \\
\dot{\hat{\mathbf{X}}}(t)=A(t) \hat{\mathbf{X}}(t)+B(t) \mathbf{U}(t)+K(t)(\mathbf{Y}(t)-C(t) \hat{\mathbf{X}}(t))
\end{array}\right.
$$

satisfies for all $t \geq s$

$$
\delta\|\tilde{\mathbf{X}}(s)\| e^{m(t-s)} \leq\|\tilde{\mathbf{X}}(t)\| \leq \eta\|\tilde{\mathbf{X}}(s)\| e^{M(t-s)}
$$

A special case of interest is when the system (9) is bounded and UCO, i.e. when there exist $a, c, \alpha_{0}$ and $T$ strictly positive such that

$$
\|A(t)\| \leq a, \quad\|C(t)\| \leq c, \quad W^{*}(t-T, t) \geq \alpha_{0} I>0
$$

Then, the following result holds.

Theorem 2: [11] A bounded system (9) is UCE by a bounded estimator if and only if it is UCO.

Keeping the last result on mind, we now study the models (5) and (6) by investigating their UCO property.

\section{UCO property of the straight-line motion model}

System (5) is trivially UCO because the reconstructibility Grammian can be readily computed as follows

$$
\Phi(t, s)=\left(\begin{array}{cc}
1 & t-s \\
0 & 1
\end{array}\right), \quad W^{*}(t-\sigma, t)=\left(\begin{array}{cc}
\sigma & -\sigma^{2} / 2 \\
-\sigma^{2} / 2 & \sigma^{3} / 3
\end{array}\right)
$$

Hence, by inspecting the eigenvalues of $W^{*}$, one obtains $W^{*}(t-\sigma, t) \geq \sigma / 4$, for all $\sigma>0$.

\section{UCO property of the curve motion model}

To system (6), consider the following input-less system which is equivalent to it from the view-point of observability ( $b_{q}$ being known)

$$
\left\{\begin{array}{l}
\dot{\phi}(t)=r(t) \theta(t)+b_{p} \\
\dot{\theta}(t)=-r(t) \phi(t) \\
\dot{b}_{p}(t)=0
\end{array}\right.
$$

with $0<r \leq|r(t)| \leq R, \quad \forall t \geq 0$.

As was proven in [9], this system is UCO, as can be determined from a differential rank criterion. Further, an estimate of the reconstructibility Grammian can be established, for sake of quantitatively estimate the decay rate of the error. This point, given by the following result, will be exploited in future investigations involving Kalman filters in particular.

Without loss of generality, $r(t)$ is assumed to be (strictly) positive. Consider the following bijective mapping (timechange)

$$
h_{t_{0}}(t)=\int_{t}^{t_{0}} r(u) d u
$$

Lemma 3: For any $t_{0}$, consider $T_{0}=t_{0}-h_{t_{0}}^{-1}(2 \pi)$, if $r(t)$ is sufficiently close to its first order (affine) Taylor expansion for $t \in\left[t_{0}-T_{0} \ldots t_{0}\right]$, then the reconstructibility Grammian satisfies

$$
W^{*}\left(t_{0}-T_{0}, t_{0}\right) \geq \alpha T_{0} I
$$

where $\alpha=\min \left(1, \frac{1}{R^{2}}\right) \frac{r}{R} \frac{2-\sqrt{2}}{4}$.

Proof: For $r$ sufficiently close to its affine Taylor expansion, there exist $\left(r_{0}, r_{1}\right)$ which satisfy, for $t \in\left[t_{0}-\right.$ $\left.T_{0} \ldots t_{0}\right]$

$$
r(t)=r_{0}\left(1+r_{1}\left(t_{0}-t\right)+o\left(r_{1} T_{0}\right)\right)
$$

Consider the following new coordinates $(y, z)$

$$
y\left(h_{t_{0}}(t)\right)=-\phi(t), \quad z\left(h_{t_{0}}(t)\right)=\theta(t), \quad b=b_{p}
$$

From the derivative of $h_{t_{0}}$, the derivative of the new coordinates can be obtained

$$
\begin{gathered}
\dot{h}_{t_{0}}(t)=-r(t), \\
\dot{\phi}(t)=r(t) \dot{y}\left(h_{t_{0}}(t)\right), \quad \dot{\theta}(t)=-r(t) \dot{z}\left(h_{t_{0}}(t)\right)
\end{gathered}
$$

Consider the new differential system (where we note $\tau=$ $\left.h_{t_{0}}(t)\right)$

$$
\dot{y}(\tau)=z(\tau)+\frac{b}{r\left(h_{t_{0}}^{-1}(\tau)\right)}, \quad \dot{z}(\tau)=-y(\tau), \quad \dot{b}(\tau)=0
$$

For any $\mathbf{X}_{2}\left(t_{0}\right)=\left[\begin{array}{lll}\phi\left(t_{0}\right) & \theta\left(t_{0}\right) & b_{p}\end{array}\right]_{0}^{T}$, equivalent initial conditions can be easily found

$$
\phi\left(t_{0}\right)=-y_{0}, \quad \theta\left(t_{0}\right)=z_{0}, \quad b_{p}=b_{0}
$$

System (14) is a forced linear time invariant oscillator of the form

$$
\ddot{z}(\tau)=-z(\tau)+f(\tau)
$$

Its solution is as follows

$z(\tau)=z_{0} \cos (\tau)-y_{0} \sin (\tau)+\int_{0}^{\tau} \sin (\tau-\sigma) f(\sigma) d \sigma$ 
Then, one can estimate the Grammian

$$
\begin{aligned}
& X_{2}^{T}\left(t_{0}\right) W^{*}\left(t_{0}-T_{0}, t_{0}\right) X_{2}\left(t_{0}\right) \\
& =X_{2}^{T}\left(t_{0}\right) \int_{t_{0}-T_{0}}^{t_{0}} \Phi_{2}^{T}\left(t, t_{0}\right) C_{2}^{T}(t) C_{2}(t) \Phi_{2}\left(t, t_{0}\right) d t X_{2}\left(t_{0}\right) \\
& =\int_{t_{0}-T_{0}}^{t_{0}} X_{2}^{T}(s) C_{2}^{T} C_{2} X_{2}(s) d s=\int_{t_{0}-T_{0}}^{t_{0}} \theta^{2}(s) d s
\end{aligned}
$$

To lower-bound (16), from the Taylor expansion of $r(t)$ (Eq.(13)) which assumes that $r$ is close to its affine Taylor expansion, one can compute

$$
\begin{aligned}
& f(\tau)=-\frac{b}{r\left(h_{t_{0}}^{-1}(\tau)\right)}=f_{0}\left(1+f_{1} \tau+o\left(f_{1} T_{0}^{\prime}\right)\right) \\
& \text { with } \begin{cases}f_{0}=-\frac{b}{r_{0}} & f_{1}=\frac{r_{1}}{r_{0}} \\
T_{0}^{\prime}=h_{t_{0}}\left(t_{0}-T_{0}\right)=2 \pi & f_{1} T_{0}^{\prime} \simeq r_{1} T_{0}\end{cases}
\end{aligned}
$$

Introducing the Taylor expansion of (17) in the solution (15), one obtains

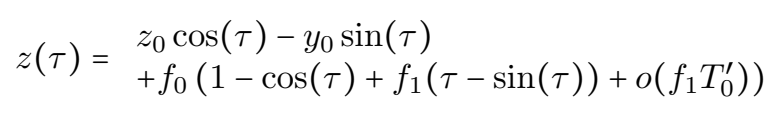

Now, consider the following decomposition of the integral

$$
\int_{0}^{T_{0}^{\prime}} z^{2}(\tau) d \tau=I_{1}+I_{2}+I_{3}+I_{4}+I_{5}+I_{6}
$$

Each term can be computed separately

$$
\begin{aligned}
& I_{1}=\int_{0}^{T_{0}^{\prime}}\left(z_{0} \cos (\tau)\right)^{2} d \tau=\frac{z_{0}^{2}}{2} T_{0}^{\prime} \\
& I_{2}=\int_{0}^{T_{0}^{\prime}}\left(y_{0} \sin (\tau)\right)^{2} d \tau=\frac{y_{0}^{2}}{2} T_{0}^{\prime} \\
& I_{3}=\int_{0}^{T_{0}^{\prime}} f_{0}^{2}\left(\begin{array}{c}
1-\cos (\tau) \\
+f_{1}(\tau-\sin (\tau))+o\left(f_{1} T_{0}^{\prime}\right)
\end{array}\right)^{2} d \tau \\
& =\frac{3}{2} f_{0}^{2} T_{0}^{\prime}\left(1+\frac{2}{3} f_{1} T_{0}^{\prime}++o\left(f_{1} T_{0}^{\prime}\right)\right) \\
& I_{4}=-2 \int_{0}^{T_{0}^{\prime}} z_{0} \cos (\tau) y_{0} \sin (\tau) d \tau=0 \\
& I_{5}=2 \int_{0}^{T_{0}^{\prime}} z_{0} \cos (\tau) f_{0}\left(\begin{array}{c}
1-\cos (\tau) \\
+f_{1}(\tau-\sin (\tau)) \\
+o\left(f_{1} T_{0}^{\prime}\right)
\end{array}\right) d \tau \\
& =-z_{0} f_{0} T_{0}\left(1+o\left(f_{1} T_{0}^{\prime}\right)\right) \\
& I_{6}=-2 \int_{0}^{T_{0}^{\prime}} y_{0} \sin (\tau) f_{0}\left(\begin{array}{c}
1-\cos (\tau) \\
+f_{1}(\tau-\sin (\tau)) \\
+o\left(f_{1} T_{0}^{\prime}\right)
\end{array}\right) d \tau \\
& =3 y_{0} f_{0}\left(f_{1} T_{0}^{\prime}+o\left(f_{1} T_{0}^{\prime}\right)\right)
\end{aligned}
$$

Gathering the expressions above, a symmetric matrix $\Pi$ can be constructed such that

$$
\int_{0}^{T_{0}^{\prime}} z^{2}(\tau) d \tau=\frac{T_{0}^{\prime}}{2}\left[\begin{array}{lll}
y_{0} & z_{0} & f_{0}
\end{array}\right] \Pi\left[\begin{array}{c}
y_{0} \\
z_{0} \\
f_{0}
\end{array}\right]
$$

with $\Pi=\left(\begin{array}{ccc}1 & 0 & 3\left(f_{1}+o\left(f_{1}\right)\right) \\ 0 & 1 & -\left(1+o\left(f_{1} T_{0}^{\prime}\right)\right) \\ \text { sym } & \text { sym } & 3+2 f_{1} T_{0}^{\prime}+o\left(f_{1} T_{0}^{\prime}\right)\end{array}\right)$
The minimum eigenvalue can be bounded thanks to the positiveness of $f_{1}$

$$
\lambda_{\min }(\Pi)=\left(2+f_{1} T_{0}^{\prime}-\sqrt{2+2 f_{1} T_{0}^{\prime}}+o\left(f_{1} T_{0}^{\prime}\right)\right) \geq 1 / 2
$$

Thus, the Grammian (16) can be lower-bounded

$$
\begin{aligned}
\int_{t_{0}-T_{0}}^{t_{0}} \theta^{2}(t) d t & =\int_{t_{0}-T_{0}}^{t_{0}} z^{2}\left(h_{t_{0}}(t)\right) d t \\
& =\int_{0}^{T_{0}^{\prime}} z^{2}(\tau) \frac{d \tau}{r\left(h_{t_{0}}^{-1}(\tau)\right)} \geq \frac{1}{R} \int_{0}^{T_{0}^{\prime}} z^{2}(\tau) d \tau \\
& \geq \frac{r}{R} \frac{T_{0}}{4}\left(y_{0}^{2}+z_{0}^{2}+f_{0}^{2}\right) \\
& \geq \min \left(1, \frac{1}{r_{0}^{2}}\right) \frac{r}{R} \frac{T_{0}}{4}\left\|\mathbf{X}_{2}\left(t_{0}\right)\right\|^{2}
\end{aligned}
$$

Then, with $\alpha=\min \left(1, \frac{1}{R^{2}}\right) \frac{r}{4 R}$, we have proven

$$
W^{*}\left(t_{0}-T_{0}, t_{0}\right) \geq \alpha T_{0} I
$$

Further, one can establish the following result.

Proposition 1: For all $t_{0}$, for all $T \geq \frac{2 \pi}{r}$, if there exist $\left(r_{0}, r_{1}\right)$ such that, for $t \in\left[t_{0}-\frac{2 \pi}{r} \ldots t_{0}\right]$,

$$
r(t)=r_{0}\left(1+r_{1}\left(t_{0}-t\right)+o\left(\frac{2 \pi r_{1}}{r}\right)\right)
$$

then the reconstructibility Grammian $W^{*}$ satisfies

$$
W^{*}\left(t_{0}-T, t_{0}\right) \geq \alpha \frac{2 \pi}{R} \eta I
$$

where $\alpha=\min \left(1, \frac{1}{R^{2}}\right) \frac{r}{4 R}$ and $\eta$ is the integer part of $\frac{r T}{2 \pi}$.

Proof:

With Chasles' theorem,

$$
\begin{aligned}
W^{*} & \left(t_{0}-T, t_{0}\right) \geq W^{*}\left(t_{0}-\frac{2 \pi}{r} \eta, t_{0}\right) \\
& \geq \sum_{i=0 . . \eta-1} W^{*}\left(t_{0}-\frac{2 \pi}{r}(i+1), t_{0}-\frac{2 \pi}{r} i\right) \\
& \geq \sum_{i=0 . . \eta-1} W^{*}\left(\left(t_{0}-\frac{2 \pi}{r} i\right)-\frac{2 \pi}{r},\left(t_{0}-\frac{2 \pi}{r} i\right)\right)
\end{aligned}
$$

By assumption, Lemma 3 holds for all $t_{0}^{(i)}=t_{0}-\frac{2 \pi}{r} i$, $i=0 . . \eta-1$.

$$
W^{*}\left(t_{0}^{(i)}-T_{0}^{(i)}, t_{0}^{(i)}\right) \geq \alpha T_{0}^{(i)} I
$$

Yet,

$$
\frac{2 \pi}{R}<T_{0}^{(i)}<\frac{2 \pi}{r}
$$

Hence, since the application $T \mapsto W^{*}\left(t_{0}-T, t_{0}\right)$ is increasing for all $t_{0}$

$$
W^{*}\left(t_{0}^{(i)}-\frac{2 \pi}{r}, t_{0}^{(i)}\right) \geq \alpha \frac{2 \pi}{R} I
$$

Finally,

$$
W^{*}\left(t_{0}-T, t_{0}\right) \geq \alpha \eta \frac{2 \pi}{R} I
$$

The constants $T$ and $\alpha \eta$ of UCO of the system are depending, on one hand, on the lower bound of the reconstructibility Grammian, and on the other hand, on the length 


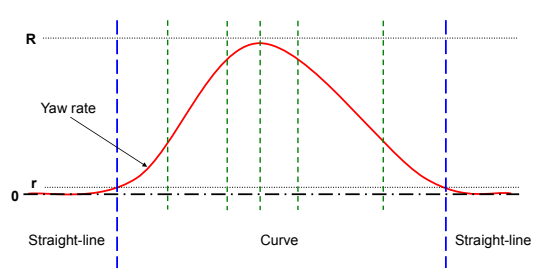

Fig. 4. Extra decomposition of the curve motion

of the integration interval in Eq. (10). To establish the proof above, the whole curve motion has been considered and the constants arise from a worst case analysis: the lower-bound is proportional to the minimum of the yaw rate divided by its maximum, and the integration length is lower-bounded by the inverse of the minimum of the yaw rate (at small values of the yaw rate, the system is poorly observable).

Yet, an extra decomposition of the curve motion can be considered as well for sake of obtaining tighter estimates. This point is illustrated in Fig. 4. The switching policy defines the curve motion as the portion between the blue lines, considering short parts of curve between each couple of green lines. First, the assumption of linear variation of the yaw rate is more easily fulfilled, and second, the central parts with high rate present a larger minimum yaw rate, yielding, in turn, an increase level of observability for a shorter integration length of the Grammian.

\section{E. UCE property of separate subsystems}

As discussed early, System (5) is UCO. Let $K_{1}(t)$ be a bounded gain given by Theorem 2 . The observer is as follows,

$$
\dot{\hat{\mathbf{X}}}_{1}=A_{1} \hat{\mathbf{X}}_{1}+B_{1} \mathbf{U}+K_{1}(t)\left(\mathbf{Y}_{1}-C_{1} \hat{\mathbf{X}}_{1}\right)
$$

At time $t_{i}+T_{1}$, estimates $\hat{\theta}(2 \mathrm{i}+1)$ and $\hat{b}_{q}(2 \mathrm{i}+1)$ are available. In particular, the value $\hat{b}_{q}(2 i+1)$ can be used to drive System (6). Since the bias $b_{q}$ is constant, considering it as a given parameter, the System (6) is also UCO (see Section IIID). Let $K_{2}(t)$ be a bounded gain given by Theorem 2 . The observer is computed as follows

$$
\dot{\hat{\mathbf{X}}}_{2}=A_{2}(t) \hat{\mathbf{X}}_{2}+B_{2} \mathbf{U}+D_{2} \hat{b}_{q}(2 \mathrm{i}+1)+K_{2}(t)\left(\mathbf{Y}_{2}-C_{2} \hat{\mathbf{X}}_{2}\right)
$$

Combining System (5) with Eq. (20) and System (6) with Eq. (21), one obtains the error dynamics

$$
\begin{aligned}
& \dot{\tilde{\mathbf{X}}}_{1}=\left(A_{1}-K_{1}(t) C_{1}\right) \tilde{\mathbf{X}}_{1} \\
& \dot{\tilde{\mathbf{X}}}_{2}=\left(A_{2}(t)-K_{2}(t) C_{2}\right) \tilde{\mathbf{X}}_{2}+D_{2} \tilde{b}_{q}(2 \mathrm{i}+1)
\end{aligned}
$$

Let $\Phi_{1}$ and $\Phi_{2}$ the transition matrices for the unforced systems corresponding to Eq. (22) and Eq. (23), respectively, one has

$$
\begin{array}{ll}
\frac{\partial \Phi_{1}}{\partial t}(t, s)=\left(A_{1}-K_{1}(t) C_{1}\right) \Phi_{1}(t, s), & \Phi_{1}(t, t)=I \\
\frac{\partial \Phi_{2}}{\partial t}(t, s)=\left(A_{2}(t)-K_{2}(t) C_{2}\right) \Phi_{2}(t, s), & \Phi_{2}(t, t)=I
\end{array}
$$

From Definiton 2 and Theorem 2, there exist $\left(k_{1}, \lambda_{1}, k_{2}, \lambda_{2}\right)$ such that

$$
\left\|\Phi_{1}(t, s)\right\| \leq k_{1} e^{-\lambda_{1}(t-s)}, \quad\left\|\Phi_{2}(t, s)\right\| \leq k_{2} e^{-\lambda_{2}(t-s)}
$$

\section{SUFFICIENT CONDITION FOR CONVERGENCE OF TIO}

Our goal is to study the convergence of $\hat{\mathbf{X}}_{I I}$, in particular from $t_{i}$ to $t_{i+1}$. We now use the estimates established above to study the convergence of the TIO structure introduced in Section II-D. The solutions of System (22) and System (23) are

$$
\begin{aligned}
& \tilde{\mathbf{X}}_{1}(2 \mathrm{i}+1)=\Phi_{1}\left(t_{i}+T_{1}, t_{i}\right) \tilde{\mathbf{X}}_{1}(2 \mathrm{i}) \\
& \tilde{\mathbf{X}}_{2}(2(\mathrm{i}+1))=\Phi_{2}\left(t_{i}+T_{1}+T_{2}, t_{i}+T_{1}\right) \tilde{\mathbf{X}}_{2}(2 \mathrm{i}+1) \\
& +\int_{0}^{T_{2}} \Phi_{2}\left(t_{i}+T_{1}+T_{2}, t_{i}+T_{1}+\tau\right) D_{2} \tilde{b}_{q}(2 \mathrm{i}+1) d \tau
\end{aligned}
$$

Combining Eq. (25) and Eq. (26) with Eq. (24), one obtains

$$
\begin{aligned}
& \left\|\tilde{\mathbf{X}}_{1}(2 \mathrm{i}+1)\right\| \leq \alpha\left\|\tilde{\mathbf{X}}_{1}(2 \mathrm{i})\right\| \\
& \left\|\tilde{\mathbf{X}}_{2}(2(\mathrm{i}+1))\right\| \leq \beta\left\|\tilde{\mathbf{X}}_{2}(2 \mathrm{i}+1)\right\|+\gamma\left\|\tilde{b}_{q}(2 \mathrm{i}+1)\right\|
\end{aligned}
$$

with $\alpha=k_{1} e^{-\lambda_{1} T_{1}}, \beta=k_{2} e^{-\lambda_{2} T_{2}}$ and $\gamma=\frac{k_{2}}{\lambda_{2}}\left(1-e^{-\lambda_{2} T_{2}}\right)$.

From Eq. (7-8), the following inequalities can be obtained

$$
\begin{aligned}
& \|\tilde{\phi}(2 \mathrm{i}+1)\|=\left\|\tilde{\phi}(2 \mathrm{i})+T_{1} \tilde{b}_{p}(2 \mathrm{i})\right\| \leq\|\tilde{\phi}(2 \mathrm{i})\|+T_{1}\left\|\tilde{b}_{p}(2 \mathrm{i})\right\| \\
& \left\|\tilde{b}_{p}(2 \mathrm{i}+1)\right\|=\left\|\tilde{b}_{p}(2 \mathrm{i})\right\|, \quad\left\|\tilde{b}_{q}(2(\mathrm{i}+1))\right\|=\left\|\tilde{b}_{q}(2 \mathrm{i}+1)\right\|
\end{aligned}
$$

To establish further estimates on $\tilde{\mathbf{X}}_{1}$ and $\tilde{\mathbf{X}}_{2}$, we consider new variables. Let vectors $\mathbf{Z}_{i}, i=0 . .5$ be defined as follows

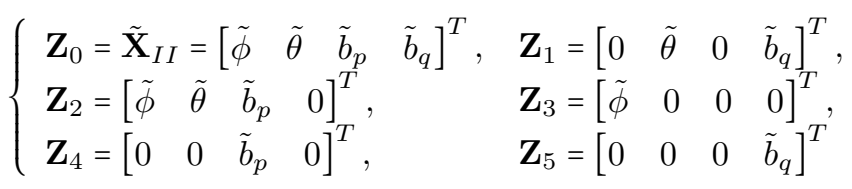

With these notations, the following equations hold

$$
\begin{gathered}
\left\|\mathbf{Z}_{0}\right\|^{2}=\left\|\mathbf{Z}_{1}\right\|^{2}+\left\|\mathbf{Z}_{3}\right\|^{2}+\left\|\mathbf{Z}_{4}\right\|^{2}=\left\|\mathbf{Z}_{2}\right\|^{2}+\left\|\mathbf{Z}_{5}\right\|^{2} \\
\left\|\mathbf{Z}_{i}\right\| \leq\left\|\mathbf{Z}_{0}\right\|, \quad i=0 . .5
\end{gathered}
$$

Equivalence with $\tilde{\mathbf{X}}_{1}$ and $\tilde{\mathbf{X}}_{2}$ are also available

$$
\begin{gathered}
\left\|\mathbf{Z}_{1}\right\|=\left\|\tilde{\mathbf{X}}_{1}\right\|,\left\|\mathbf{Z}_{2}\right\|=\left\|\tilde{\mathbf{X}}_{2}\right\|, \\
\left\|\mathbf{Z}_{3}\right\|=\|\tilde{\phi}\|,\left\|\mathbf{Z}_{4}\right\|=\left\|\tilde{b}_{p}\right\|,\left\|\mathbf{Z}_{5}\right\|=\left\|\tilde{b}_{q}\right\|
\end{gathered}
$$

Eq. $(27,28,29,30)$ are re-written as follows

$$
\begin{gathered}
\left\|\mathbf{Z}_{1}(2 \mathrm{i}+1)\right\|^{2} \leq \alpha^{2}\left\|\mathbf{Z}_{1}(2 \mathrm{i})\right\|^{2} \\
\left\|\mathbf{Z}_{2}(2(\mathrm{i}+1))\right\|^{2} \leq\left(\beta\left\|\mathbf{Z}_{2}(2 \mathrm{i}+1)\right\|+\gamma\left\|\mathbf{Z}_{5}(2 \mathrm{i}+1)\right\|\right)^{2} \\
\left\|\mathbf{Z}_{3}(2 \mathrm{i}+1)\right\|^{2} \leq\left(\left\|\mathbf{Z}_{3}(2 \mathrm{i})\right\|+T_{1}\left\|\mathbf{Z}_{4}(2 \mathrm{i})\right\|\right)^{2} \\
\left\|\mathbf{Z}_{4}(2 \mathrm{i}+1)\right\|^{2}=\left\|\mathbf{Z}_{4}(2 \mathrm{i})\right\|^{2} \\
\left\|\mathbf{Z}_{5}(2(\mathrm{i}+1))\right\|^{2}=\left\|\mathbf{Z}_{5}(2 \mathrm{i}+1)\right\|^{2}
\end{gathered}
$$

From Eq. $(31,35,37,38)$,

$$
\begin{array}{rl|l}
\left\|\mathbf{Z}_{0}(2 \mathrm{i}+1)\right\|^{2} \leq & \begin{array}{c}
\alpha^{2}\left\|\mathbf{Z}_{1}(2 \mathrm{i})\right\|^{2}+\left\|\mathbf{Z}_{4}(2 \mathrm{i})\right\|^{2} \\
+\left(\left\|\mathbf{Z}_{3}(2 \mathrm{i})\right\|+T_{1}\left\|\mathbf{Z}_{4}(2 \mathrm{i})\right\|\right)^{2}
\end{array} \\
\leq \mid \begin{array}{c}
\alpha^{2}\left\|\mathbf{Z}_{1}(2 \mathrm{i})\right\|^{2}+\left(1+T_{1}^{2}\right)\left\|\mathbf{Z}_{4}(2 \mathrm{i})\right\|^{2} \\
+\left\|\mathbf{Z}_{3}(2 \mathrm{i})\right\|^{2}+2 T_{1}\left\|\mathbf{Z}_{3}(2 \mathrm{i})\right\|\left\|\mathbf{Z}_{4}(2 \mathrm{i})\right\|
\end{array}
\end{array}
$$


With Eq. (31,32),

$$
\begin{aligned}
\left\|\mathbf{Z}_{0}(2 \mathrm{i}+1)\right\|^{2} & \leq \max \left(\alpha^{2}, 1+T_{1}^{2}\right)\left\|\mathbf{Z}_{0}(2 \mathrm{i})\right\|^{2}+2 T_{1}\left\|\mathbf{Z}_{0}(2 \mathrm{i})\right\|^{2} \\
& \leq \max \left(\alpha^{2}+2 T_{1},\left(1+T_{1}\right)^{2}\right)\left\|\mathbf{Z}_{0}(2 \mathrm{i})\right\|^{2}
\end{aligned}
$$

From Eq. (31,36,39),

$$
\begin{aligned}
\left\|\mathbf{Z}_{0}(2(\mathrm{i}+1))\right\|^{2} & \leq\left(\beta\left\|\mathbf{Z}_{2}(2 \mathrm{i}+1)\right\|+\gamma\left\|\mathbf{Z}_{5}(2 \mathrm{i}+1)\right\|\right)^{2}+\left\|\mathbf{Z}_{5}(2 \mathrm{i}+1)\right\|^{2} \\
& \leq \mid \begin{array}{c}
\beta^{2}\left\|\mathbf{Z}_{0}(2 \mathrm{i}+1)\right\|^{2}+\left(1+\gamma^{2}-\beta^{2}\right)\left\|\mathbf{Z}_{5}(2 \mathrm{i}+1)\right\|^{2} \\
+2 \beta \gamma\left\|\mathbf{Z}_{2}(2 \mathrm{i}+1)\right\|\left\|\mathbf{Z}_{5}(2 \mathrm{i}+1)\right\|
\end{array}
\end{aligned}
$$

Knowing that $\left\|\mathbf{Z}_{5}(2 \mathrm{i}+1)\right\| \leq\left\|\mathbf{Z}_{1}(2 \mathrm{i}+1)\right\|$, with Eq. $(35,32)$,

$$
\begin{aligned}
\left\|\mathbf{Z}_{0}(2(\mathrm{i}+1))\right\|^{2} \leq & \begin{array}{c}
\left(\beta^{2}+2 \beta \gamma\right)\left\|\mathbf{Z}_{0}(2 \mathrm{i}+1)\right\|^{2} \\
+\max \left(0,1+\gamma^{2}-\beta^{2}\right)\left\|\mathbf{Z}_{1}(2 \mathrm{i}+1)\right\|^{2}
\end{array} \\
\leq & \begin{array}{c}
\left(\beta^{2}+2 \beta \gamma\right)\left\|\mathbf{Z}_{0}(2 \mathrm{i}+1)\right\|^{2} \\
+\alpha^{2} \max \left(0,1+\gamma^{2}-\beta^{2}\right)\left\|\mathbf{Z}_{1}(2 \mathrm{i})\right\|^{2}
\end{array}
\end{aligned}
$$

Combining these with Eq. $(40,32)$, one obtains

$\frac{\left\|\mathbf{Z}_{0}(2(\mathrm{i}+1))\right\|^{2}}{\left\|\mathbf{Z}_{0}(2 \mathrm{i})\right\|^{2}} \leq \mid \begin{gathered}\left(\beta^{2}+2 \beta \gamma\right) \max \left(\alpha^{2}+2 T_{1},\left(1+T_{1}\right)^{2}\right) \\ +\alpha^{2} \max \left(0,1+\gamma^{2}-\beta^{2}\right)\end{gathered}$

Note $\Gamma=\left(\beta^{2}+2 \beta \gamma\right) \max \left(\alpha^{2}+2 T_{1},\left(1+T_{1}\right)^{2}\right)+$ $\alpha^{2} \max \left(0,1+\gamma^{2}-\beta^{2}\right)$. From the construction of $\alpha, \beta$ and $\gamma$,

$$
\begin{gathered}
\lim _{T_{1} \rightarrow+\infty} \alpha=0, \quad \lim _{T_{2} \rightarrow+\infty} \beta=0, \quad \lim _{T_{2} \rightarrow+\infty} \gamma=\frac{k_{2}}{\lambda_{2}} \\
\lim _{\substack{T_{1} \rightarrow+\infty \\
T_{2} \rightarrow+\infty}} \Gamma=\lim _{\substack{T_{1} \rightarrow+\infty \\
T_{2} \rightarrow+\infty}} 2 \frac{k_{2}^{2}}{\lambda_{2}} T_{1}^{2} e^{-\lambda_{2} T_{2}}
\end{gathered}
$$

If $T_{1}=o\left(e^{\frac{\lambda_{2}}{2} T_{2}}\right)$, then

$$
\lim _{\substack{T_{1} \rightarrow+\infty \\ T_{2} \rightarrow+\infty}} \Gamma=0
$$

We can now state the following result.

Proposition 2: Consider Temporally Interconnected Observers (TIO), given by Eq. (7-8), taking the form of Eq. (2021) and illustrated in Fig. 3, used on an alternate sequence of straight-lines and curves motions of respective durations $T_{1}$ and $T_{2}$. Assume that the observers satisfy inequalities (24). Asymptotic stability of the estimates of the TIO is guaranteed for sufficiently large values of $T_{1}$ and $T_{2}$ chosen according to $T_{1}=o\left(e^{\frac{\lambda_{2}}{2} T_{2}}\right)$ where $\lambda_{2}$ is the decay rate of the curve motion observer introduced in Eq. (24).

Proof: Eq. (41) guarantees super-linear convergence (as defined in [15]) of the sequence $\left\|\mathbf{Z}_{0}(2 \mathrm{i})\right\|$ for sufficiently large times between switching. From the definition of $\mathbf{Z}_{0}$, the estimation error on $\mathbf{X}_{I I}$, taken at the discrete times $t_{i}$, converges towards zero. Since the continuous dynamics of Systems (4) is bounded, the estimation error $\left\|\tilde{\mathbf{X}}_{I I}(t)\right\|$ is asymptotically stable. From the measurements $\left(p_{m}, q_{m}\right)$ and the estimated biases, the rates $(p, q)$ can be evaluated with an error which also tends to zero. Therefore, the proposed TIO structure achieves asymptotic reconstruction of the state $\left(\begin{array}{llllll}\phi & \theta & b_{p} & b_{q} & p & q\end{array}\right)^{T}$.
For sake of illustration of the statement in Proposition 2, we consider a parametrization of $\left(T_{1}, T_{2}\right)$ that satisfy the required assumption. Consider a vector of parameter $\left(v_{1}, v_{2}\right)>$ 1 , for any $\left(T_{1}, T_{2}\right)$ such that

$$
\begin{aligned}
& T_{1}>\frac{1}{\lambda_{1}} \log \left(k_{1} \sqrt{v_{1}\left(1+\frac{k_{2}^{2}}{\lambda_{2}^{2}}\right)}\right) \\
& T_{2}>\frac{1}{\lambda_{2}} \log \left(\frac{\lambda_{2}}{\sqrt{1+\frac{\lambda_{2}^{2}}{v_{2} k_{2}^{2}\left(1+T_{1}\right)^{2}}}-1}\right)
\end{aligned}
$$

Then, the rate of convergence $\Gamma$ is bounded as follows

$$
\Gamma<\frac{1}{v_{1}}+\frac{1}{v_{2}}<1
$$

The main result of this paper is Proposition 2. It can be used as follows. Given a TIO structure, from Sections III$\mathrm{C}$ and III-D, the systems are UCO, and from Theorem 2, they are UCE. Then, there exists estimates of the form (2021) which satisfy Eq. (24) and Proposition 2 guarantees, for well-chosen sufficiently large $T_{1}, T_{2}$, the convergence of the TIO scheme. Further, quantitative estimates (24) can be obtained using Proposition 1, in connection with results on decay rates of Kalman filters. This point is the subject of future contributions. In practice, the preceding discussion must be considered in another order. The magnitudes of the measurements noises and of the dynamics uncertainties implicitly define the best possible constants $\lambda_{1}, k_{1}, \lambda_{2}, k_{2}$ in Eq. (24). Proposition 2 states, e.g. through Eq. (42-43), that under the assumption of constant biases $b_{p}, b_{q}$, the TIO scheme is asymptotically converging for sufficiently large and well chosen values of $T_{1}, T_{2}$. What can discard the application of this result to cases of practical interest is the fact that the bounds on $T_{1}$ and $T_{2}$ can be inconsistent with the assumption that the biases are constant.

\section{EXPERIMENTAL RESULTS}

We now present some results obtained on-board an actual passenger car which is equipped with a velocimeter, a barometer and MEMS gyroscopes. A GPS receiver is embedded to serve for sake of comparisons only. In the figures, we note "TIO" values (in blue) estimated with Temporally Interconnected Observers and refer to debiased values (in red) with the term "ZUPT" for Zero Velocity Update (see [1], for a detailed presentation of this reference technique).

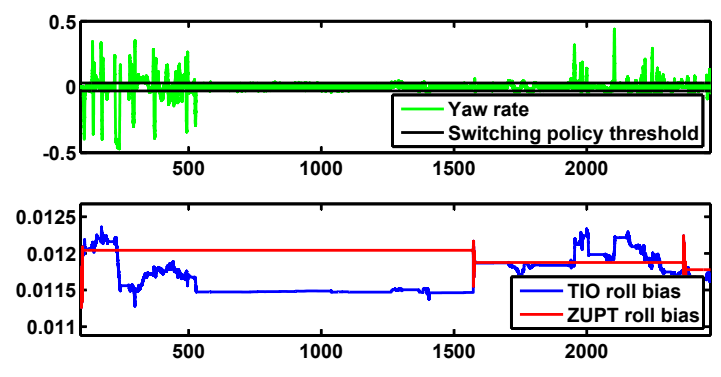

Fig. 5. Switching policy and roll bias estimation 


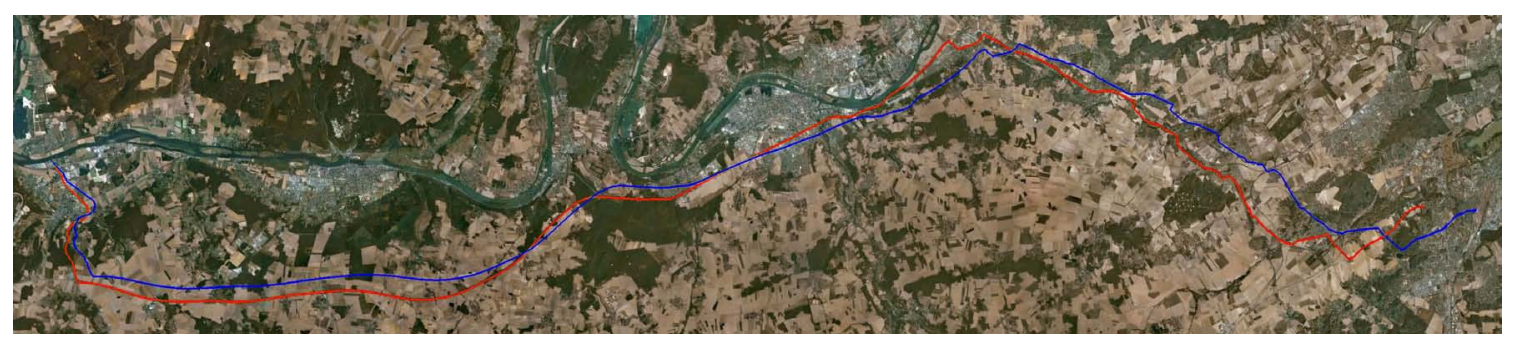

Fig. 6. Comparison of estimated trajectory (blue) with GPS (red).The approximate path length is $80 \mathrm{~km}$. obtained during a 80 min. trip.

In Figure 5, the estimated yaw rate is presented (in green) and compared to the threshold of the switching policy. In the second plot representing the roll bias estimate, a long straight line is visible with a large constant part between $500 \mathrm{~s}$ and $1500 \mathrm{~s}$, corresponding to a value of yaw rate lower than the threshold.

Figure 7 shows the pitch bias estimation which reveals the performance of the algorithm since the TIO bias is almost continuous and kindly interpolates the values estimated during ZUPTs without showing any steps like the bias estimated only during ZUPTs. Further, the TIO estimates provides continuous time estimate of the bias, while the ZUPT can only operate at discrete times (from time to time).

In Figure 7, the succession of varying values and constant parts are not easily detectable because constant parts appear only during curves which are in-between straight lines.

Figure 8 presents the heading estimation improvement due to the use of TIO. More precisely, the difference between the ZUPT heading and the TIO heading relative to the GPS are presented. In absence of an Attitude and Heading Reference System, this comparison fairly represents the improvement brought by the proposed algorithm because the heading is computed from the gyrometers, the estimated attitudes and biases. After one hour of driving, the drift of heading is reduced by 15 degrees.

The proposed method yields a significant reduction of the various biases of the sensors. Then, their information can be integrated to determine position estimates. This gives some promising results. In Figure 6, the trajectory (blue) obtained by integration of the estimated velocity along with estimated attitudes of the vehicle is presented and compared against the GPS information (red). After 80 minutes and 80 kilometers of driving, the heading error is less than 10 degrees and position error is less than 2.5 kilometers. These results have been obtained using solely the mentioned low-cost sensors: a MEMS altimeter, a velocimeter, MEMS inertial sensors. Interestingly, these results are accurate enough to serve as inputs to map-matching techniques [16], which can now be integrated in such low-cost GPS-free navigation system.

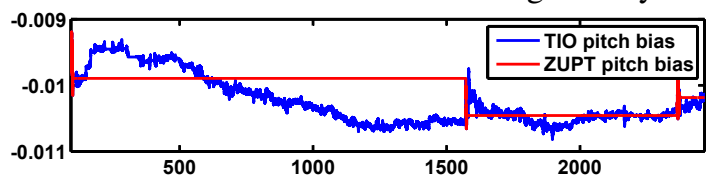

Fig. 7. Pitch bias estimation showing the continuity of the estimation Acknowledgments: The authors are indebted to Laurent Praly for fruitful discussions and insightful remarks.

\section{REFERENCES}

[1] D. A. Grejner-Brzezinska, Y. Yi, and C. K. Toth, "Bridging gps gaps in urban canyons: the benefits of zupts," J. Navigation, vol. 48, no. 4, pp. 217-225, 2001.

[2] L. Ojeda and J. Borenstein, "Non-gps navigation for security personnel and first responders," J. Navigation, vol. 60, no. 3, pp. 391-407, 2007.

[3] S. Hicks, "Advanced cruise missile guidance system description," in Proc. IEEE National Aerospace and Electronics Conference, 1993.

[4] V. Ekütekin, "Navigation and control studies on cruise missiles," Ph.D. dissertation, Middle East Technical University, 2007.

[5] S. M. Bezick, A. J. Pue, and C. M. Patzelt, "Inertial navigation for guided missile systems," John Hopkins APL Technical Digest, vol. 28, no. 4, pp. 331-342, 2010.

[6] M. Uliana, F. Andreucci, and B. Papalia, "The navigation system of an autonomous underwater vehicle for antarctic exploration," in Proc. IEEE Marine Technology Society Conference, vol. 1, 1997, pp. 403408 .

[7] S.-B. Kim, S.-Y. Lee, T.-H. Hwang, and K.-H. Choi, "An advanced approach for navigation and image sensor integration for land vehicle navigation," in Proc. IEEE 60th Vehicular Technology Conference, vol. 6, 2004, pp. 4075-4078.

[8] I. Skog and P. Handel, "In-car positioning and navigation technologies: a survey," IEEE Trans. Intelligent Transportation Systems, vol. 10, no. 1, pp. 4-21, 2009.

[9] P.-J. Bristeau, N. Petit, and L. Praly, "Design of a navigation filter by analysis of local observability," in Proc. 49th IEEE Conference on Decision and Control, 2010.

[10] G. Besançon and H. Hammouri, "On observer design for interconnectd systems," J. Mathematical Systems, Estimation, and Control, vol. 8, no. 3, pp. 1-25, 1998.

[11] M. Ikeda, H. Maeda, and S. Kodama, "Estimation and feedback in linear time-varying systems: A deterministic theory," SIAM J. Control, vol. 13, no. 2, pp. 304-326, 1975.

[12] R. E. Kalman, "Contributions to the theory of optimal control," Boletin de la Sociedad Matematica Mexicana, vol. 5, pp. 102-119, 1960.

[13] L. M. Silverman and B. D. O. Anderson, "Controllability, observability and stability of linear systems," SIAM J. Control, vol. 6, no. 1, pp. $121-130,1968$.

[14] K. S. Tsakalis and P. A. Ioannou, Linear Time-Varying Systems: Control and Adaptation. Prentice Hall, 1993.

[15] J. Nocedal and S. J. Wright, Numerical Optimization, ser. Springer Series in Operations Research. Springer, 1999.

[16] P. Davidson, M. Vazquez, and R. Piche, "Uninterrupted portable car navigation system using gps, map and inertial sensors data," in Proc. IEEE 13th International Symposium on Consumer Electronics, 2009, pp. $836-840$.

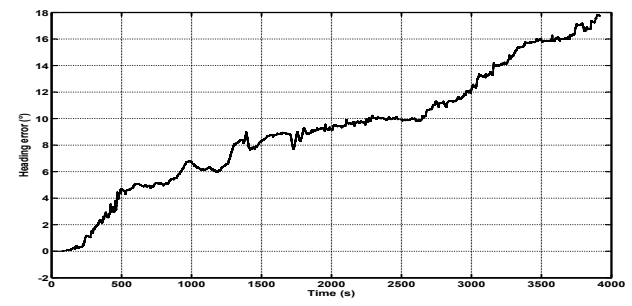

Fig. 8. Drift of ZUPT heading relative to TIO heading 